\title{
Evaporation-assisted formation of surface patterns from polymer solutions via copper tubes
}

\author{
W. Sun ${ }^{1}$, F. Q. Yang $^{2 *}$ \\ ${ }^{1}$ College of Chemistry, Chemical Engineering and Environmental Engineering Liaoning Shihua University, Fushun, \\ 113001 Liaoning, China \\ ${ }^{2}$ Materials Program, Department of Chemical and Materials Engineering University of Kentucky, KY 40506 Lexington, \\ United States
}

Received 10 January 2018; accepted in revised form 8 March 2018

\begin{abstract}
Surface patterns with controllable features are constructed via the evaporation of an acetone solution of poly (methyl methacrylate) under the confinement of a copper tube. The effects of the dimensions of copper tube and the substrate temperature on the surface patterns are systematically studied. For the substrate temperature in the range of 30 to $50^{\circ} \mathrm{C}$, the surface patterns are concentric rings consisting of waved rings and/or linked beads. The wavelength of the concentric rings decreases with the increase of the height of copper tube, and is dependent on the substrate temperature. At the substrate temperature of $20^{\circ} \mathrm{C}$, surface patterns are presented in wrinkling-like shape. At the substrate temperature of $10^{\circ} \mathrm{C}$, 'breath figure' phenomenon occurs, and 'hole network' patterns are formed. A summary of the geometrical characteristics of the surface patterns is given, which can be used to better design and control evaporation-induced surface patterns.
\end{abstract}

Keywords: coatings, evaporation, surface patterns, self-assembly, controllable features

\section{Introduction}

Surface patterns of small scales have been attracting great interests due to potential applications in a variety of areas, including electronics [1], optical devices [2], tissue engineering [3], biomedical devices $[4,5]$, energy storage $[6,7]$, etc. Various techniques have been developed to construct surface structures of small scales, such as polymer demixing [8], surface wrinkling [9], nanoimprint [10], inkjet printing [11], laser writing [12], fracture-induced pattering [13], etc. However, it is a challenge to effectively and economically construct well-ordered, controllable surface structures of small scales using most of these techniques.

Evaporation-induced patterning, which is based on the 'coffee ring' effect, is a low-cost method to form surface patterns on polymer films, micro/nano-materials, etc, [14-17]. Using pre-designed templates to control the spreading of droplets and the evaporation of solvent, well-ordered surface structures have been formed. For example, well-ordered concentric-ring patterns from different materials were formed using a 'sphere-on-flat' template $[18,19]$. The wavelength and amplitude of the formed concentric rings generally decrease with the decrease of the distance to the sphere center. Similarly, parallel stripes were constructed by the evaporation of solvent, which is confined by a 'cylinder-on-flat' template and a 'wedge-on-flat' template [20, 21]. There are also reports on the formation of surface patterns of gradients using templates without controlling the evaporation of solvent [22]. To use the principle of evaporation-induced patterns to form surface patterns of single wavelength, one needs to carefully control the evaporation of solvent or the motion of threephase contact line (CL). For example, a 'roll-based 
evaporation-induced' strategy has been used to construct parallel stripes of single wavelength by rotating a cylindrical roll to control the CL motion [23]. To improve the evaporation-induced patterning technique and construct tunable patterns, various processing parameters need to be carefully studied, including template [24], solution concentration [25], non-volatile solute [18], solvent [26], surface tensions [27], and evaporation rate, in which the evaporation rate likely is one of the important parameters controlling the formation of surface patterns. Generally, the simplest method to control the evaporation rate is to control the system temperature. Using a copper ring as a template, Sun and Yang [28] demonstrated the dependence of the geometrical characteristics of concentric rings on the substrate temperature. Sun and Yang [29] also observed evaporationinduced formation of honeycomb patterns via the 'Breath Figure' mechanism at a low temperature, which is associated with the condensation of droplets on the surface of immiscible liquid [30].

Kelly-Zion et al. [31] studied the evaporation rate of a volatile droplet within different geometries, and showed that using a 'well-geometry' can significantly reduce the evaporation rate of a volatile droplet. Their results suggest the dependence of the evaporation rate of volatile solutions on the geometrical configuration of templates. However, there is little study focusing on the dimensional effect of templates on the morphological characteristics of the surface patterns induced by evaporation.

In this study, we use copper tubes of different sizes as templates to confine polymer solutions for the construction of surface patterns via free evaporation of the solvent in the polymer solutions. The effect of the substrate temperature in a range of 0 to $50^{\circ} \mathrm{C}$ on the geometrical characteristics of the surface patterns is also studied.

\section{Materials and methods}

\subsection{Materials}

PMMA (poly(methyl methacrylate) $\left(M_{\mathrm{w}}=35000\right)$ and acetone $(99.5 \%)$ were obtained from Fisher Scientific (Pittsburgh, PA, USA). Glass slides $\left(20 \times 15 \times 1 \mathrm{~mm}^{3}\right)$ were cleaned ultrasonically with acetone for five times and then rinsed with DI water. Templates of hollow, cylindrical tubes of various dimensions were made from a copper sheet of $0.1 \mathrm{~mm}$ in thickness.

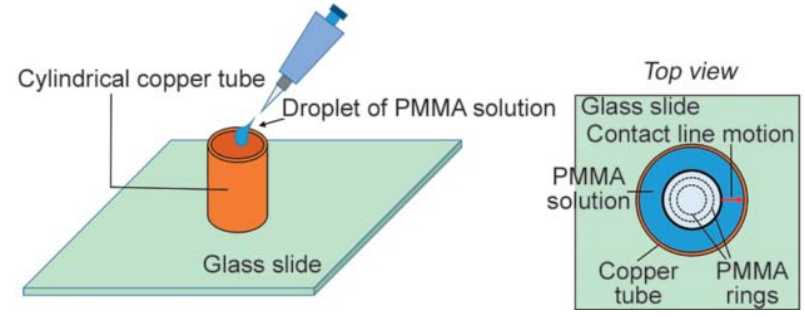

Figure 1. Schematic of experimental setup.

\subsection{Evaporation-induced surface patterning}

Acetone solution of $0.1 \mathrm{wt} \%$ PMMA in concentration was prepared by dissolving PMMA in acetone. Figure 1 schematically shows the experimental setup. A hollow-cylindrical tube was placed on the surface of a glass slide with the flank perpendicular to the surface of the glass slide. A droplet of the polymer solution, which was dripped into the hollow-cylindrical tube, spread on the surface of the glass slide under the confinement of the hollow-cylindrical tube. Hollowcylindrical tubes of different heights $(1,3,6$, and $9 \mathrm{~mm})$ and inner diameters $(2.5,4.5$, and $6.5 \mathrm{~mm})$ were used to study the effect of the tube dimensions. To have approximately the same amount of polymer per unit area on the glass slides in the hollow-cylindrical tubes, the droplet volume was $6.0 \mu \mathrm{L}$ for the inner diameter of $2.5 \mathrm{~mm}, 19.4 \mu \mathrm{L}$ for the inner diameter of $4.5 \mathrm{~mm}$, and $40.6 \mu \mathrm{L}$ for the inner diameter of $6.5 \mathrm{~mm}$. A thermal stage was used to control the evaporation of the polymer solution in a hollow-cylindrical tube in a temperature range of 0 to $50^{\circ} \mathrm{C}$. The surface morphology of the formed surface patterns was characterized using an optical microscope (Nikon ECLIPSE LV100POL, Japan), and analyzed by Imagepro plus software. It is worth mentioning that atomic force microscopy (AFM), which is an effective technique to analyze 3 -dimentional topology, is not suitable for this work, since the height of the structures is beyond the limit of AFM $(3 \mu \mathrm{m})$. Thus only 2-dimensional features were investigated in this study.

\section{Simple analysis for formation of concentric rings}

Figure 2 shows schematically the evaporation in a copper tube and the forces acting on the CL. With the spreading of a droplet of the polymer solution on the surface of a glass slide, the droplet rapidly covers the surface of the glass slide confined in a copper tube, which leads to the formation of a concave interface between the polymer solution and air due to 


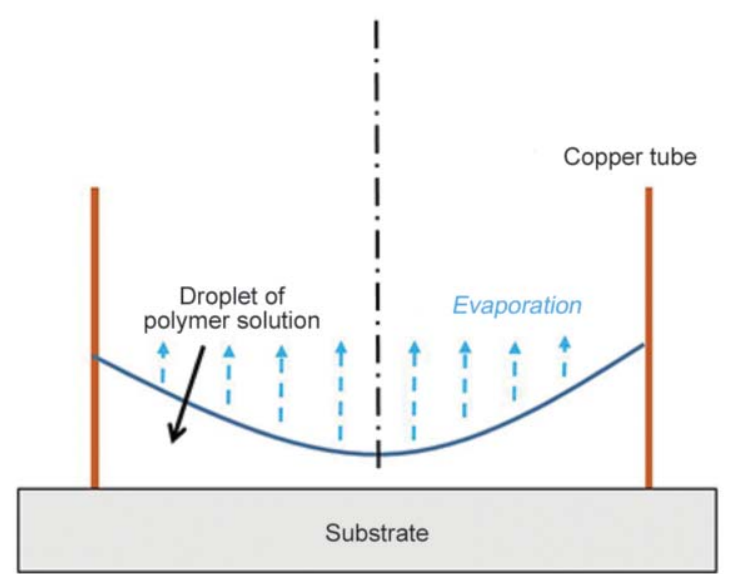

a)

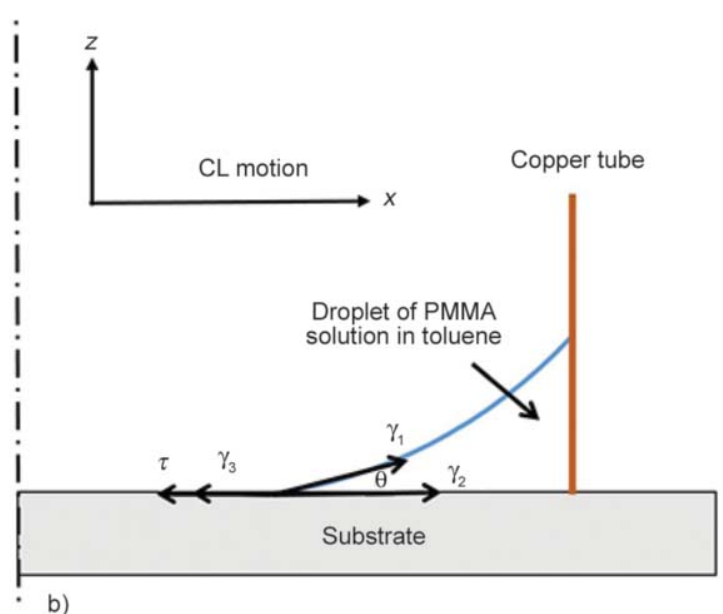

b)

Figure 2. Schematic of (a) the evaporation in a copper tube, and (b) the forces acting on the CL.

the capillary effect on the wetting between the polymer solution and the inner surface of the copper tube and a layer of the polymer solution sandwiched between the interface and the glass slide (Figure 2a). The evaporation of the volatile solvent (acetone) in the polymer solution causes the morphological change of the interface and reduces the layer thickness of the polymer solution.

Continuous evaporation of the volatile solvent (acetone) causes gradual decrease of the layer thickness of the polymer solution on the glass slide, and an inner contact line appears at the center when a criti$\mathrm{cal}$ volume is reached. Figure $2 \mathrm{~b}$ illustrates the forces acting on the CL during the evaporation of the solvent. The Equation (1):

$\rho \frac{\mathrm{d}^{2} x}{\mathrm{~d} t^{2}}=\gamma_{1} \cos \theta+\gamma_{2}-\gamma_{3}-\tau$

where $\rho$ is the line density of mass of the contact line, $t$ is time, $\gamma_{1}$ is the surface tension between toluene and air, $\gamma_{2}$ is the surface tension between toluene and the PMMA, $\gamma_{3}$ is the surface tension between air and PMMA, $\theta$ is the dynamic contact angle of the polymer solution between the glass slide and air, and $\tau$ is the resistance per unit exerted on the contact line. The variation of the contact angle is dependent on the motion of the $\mathrm{CL}$ and the evaporation of the solvent with the receding of the CL causing the increase of the dynamic contact angle $\theta$, and the solvent evaporation leading to the decrease of the dynamic contact angle $\theta$. With the formation of the CL, the CL starts to move towards the inner surface of the copper tube accompanying with the increase of the dynamic contact angle $\theta$. When the dynamic contact angle $\theta$ reaches the 'critical value' of $\cos \theta=\left(\gamma_{3}+\tau-\gamma_{2}\right) / \gamma_{1}$, the CL starts to decelerate and eventually reaches a 'dynamic' stick state to form a concentric ring. Note that each individual polymer ring corresponds to a 'dynamic' stick state associated with the accumulation of polymer in the vicinity of the CL [15]. During the 'dynamic' stick state, the resultant force on the ring is locally at a quasi-static state; there exists uneven evaporation of solvent over the surface of the layer of the polymer solution, which introduces a concentration gradient, leading to a convective flow and the accumulation of polymer in the vicinity of the CL. Continuous evaporation of solvent causes the decrease of the contact angle, and eventually there is $\gamma_{1} \cos \theta+\gamma_{2}-\gamma_{3}>\tau$. The CL starts to move again until it reaches another 'dynamic' stick state to form another concentric ring. The stick-slip motion of the CL eventually leads to the formation of concentricring patterns. Note that the dynamic contact angle $\theta$ and the evaporation rate are dependent on the geometry and dimensions of the template.

\section{Results and discussion}

Figure 3 shows typical optical images of the surface pattern formed via the evaporation of a droplet of the PMMA solution confined within a copper tube of $2.5 \mathrm{~mm}$ in inner diameter and $1 \mathrm{~mm}$ in height at a substrate temperature of $50^{\circ} \mathrm{C}$. It is evident that concentric-ring pattern was formed over a large area during the evaporation of the droplet, as shown in Figure 2a. The concentric rings covered almost the entire area inside the copper tube. There is a featureless zone within the innermost ring, which is much smaller than that formed via the evaporation of a droplet of the PMMA solution confined by a thin copper ring and similar experimental parameters 


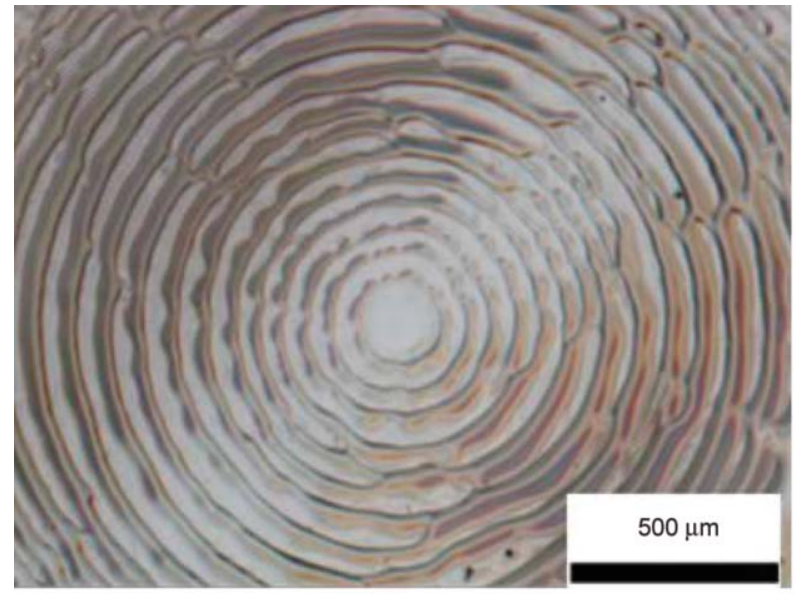

a)

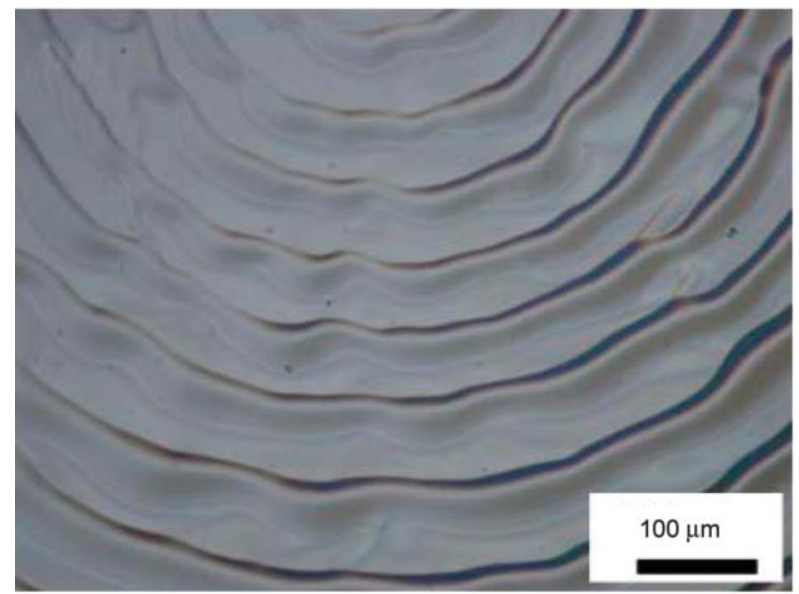

c)

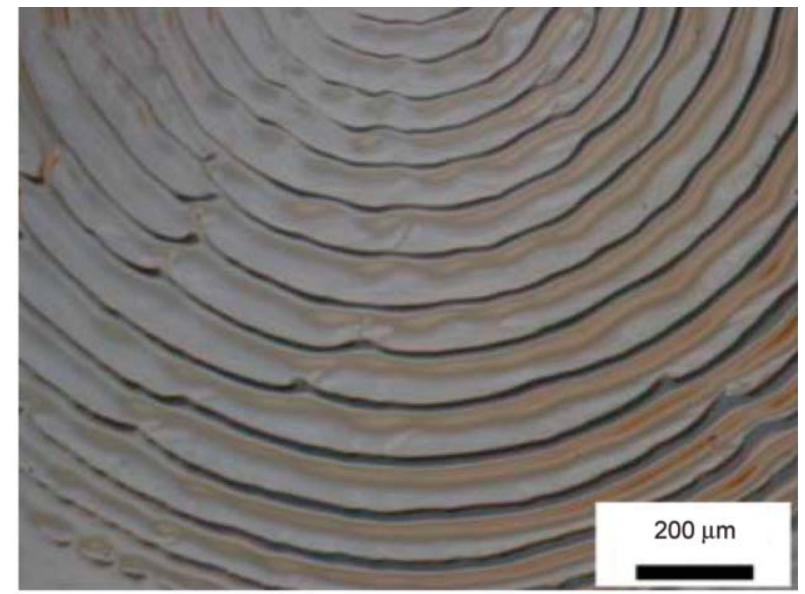

b)

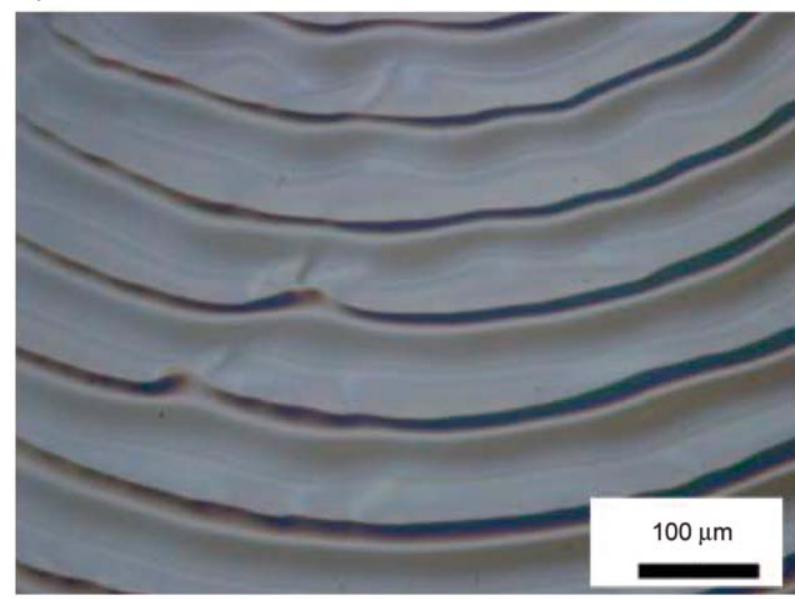

d)

Figure 3. Optical images of concentric-rings formed with a hollow-cylindrical tube at a substrate temperature of $50^{\circ} \mathrm{C}$ (inner diameter: $2.5 \mathrm{~mm}$, height: $1 \mathrm{~mm}$ ); (a) an overview of the surface patterns around the center, and (b-d) enlarged views of the concentric rings.

[32]. As discussed above, the formation of the concentric rings is attributed to the 'stick-slip' motion of the '3-phase contact line'.

From Figure 3b-3d, one can note that the concentric rings are present in a wave-like shape instead of smooth rings. Such a wave-like shape reveals the presence of local instability during the CL motion, which can be attributed to local disturbance induced by the evaporation of the polymer solution. Conventionally, the evaporation-induced instabilities is due to the surface-tension-induced Bénard-Marangoni convection [34], which depends on the temperature gradient. However, De Gennes [35] proposed that the concentration gradient usually plays a more important role than the thermal convection in the occurrence of the instabilities for the evaporation of a polymer solution film. From qualitative scaling analysis, De Gennes [35] arrived at three main conclusions: (a) the concentration gradient in a non-glassy polymer film is expected to induce convective instabilities when the film thickness is larger than a critical value; (b) the instabilities take place early, when the solution in the film is still dilute; (c) the instabilities would introduce surface roughness or certain patterns. De Gennes [36] also suggested that a glassy film may first form near free surface which is under the action of mechanical tension, and cracks or other patterns can then form during the relaxation of the tension. Bormashenko and coworkers [37, 38] demonstrated that 'mass transport' is the main mechanism controlling the formation of microscale instabilities, rather than the 'Bénard-Marangoni convection' from both experimental and theoretical work. The instability mechanisms for the formation of the wave-like shape is much more complicated, and likely consist in the combination effects of the concentration gradient and the 'mass transport' in the film.

The wavelength of the surface patterns of concentric rings is defined as the distance between two adjacent polymer rings (Figure 3c). From Figure 3, it seems 
that the wavelength does not change significantly with the decrease of the distance to the inner surface of the copper tube. Such a trend is different from the concentric rings formed within a circular-ring template made of a copper wire or other templates with free evaporation $[21,33,39]$, which suggests the effect of the copper tube on the CL motion with the approximately same 'receding' distance for each 'stick-slip' cycle.

\subsection{Effect of the dimensions of copper tube}

Figure $4 \mathrm{a}-\mathrm{d}$ shows optical images of the concentric rings formed via the evaporation of a droplet of the acetone solution with $0.1 \mathrm{wt} \%$ PMMA under the confinement of copper tubes of different heights. The inner diameter of the copper tubes was $2.5 \mathrm{~mm}$, and the substrate temperature was $50^{\circ} \mathrm{C}$. It is evident that concentric rings were formed on the surfaces of the glass slides for all the copper cubes with the heights in the range of $1 \mathrm{~mm}$ to $9 \mathrm{~mm}$, while the concentric rings exhibit different characteristics. The concentric rings formed with the copper tube of $\sim 1 \mathrm{~mm}$ in height is present in a wave-like shape (Figure $4 \mathrm{a}$ ), and the concentric rings formed with the copper tube of $\sim 9 \mathrm{~mm}$ in height consist of discrete and/or linked beads. The presence of the beads in the concentric rings reveals the evolution of local surface instability driven by surface tension. It is known that a straight, cylindrical thread is unstable, and any local perturbation can lead to the disintegration of the thread and the formation of multiple droplets due to the minimization of surface energy. Such behavior suggests that there are multiple metastable states for the formed concentric rings, which depend on the evaporation behavior of the acetone in the solution. The height of the copper tube plays an important role in the confinement effect of the copper tube on the evaporation of the acetone in the polymer solution.

Figure $4 \mathrm{~b}$ and $4 \mathrm{c}$ reveals the transition of the wavelike rings to the rings with linked beads for the copper tubes with 3 and $6 \mathrm{~mm}$ in height, respectively. The surface patterns formed with the copper tube of $6 \mathrm{~mm}$ in height more resemble the patterns formed with the copper tube of $9 \mathrm{~mm}$ in height, and the surface patterns formed with the copper tube of $3 \mathrm{~mm}$ in height more resemble the patterns formed with the copper tube of $1 \mathrm{~mm}$ in height. The surface patterns gradually evolve from the wave-like rings to the rings with linked-beads as the distance to the inner surface of the copper tube increases. Such a trend suggests that the surface patterns are a function of local evaporation rate, which depends on the height of the copper tube. From Figure $4 c-d$, one can note again that there is no dramatic change of the wavelength with the distance to the inner surface of the copper tube for individual surface patterns. The average wavelength of the concentric rings decreases with the increase of the height of the copper tube.

Figure $4 \mathrm{c}, 4 \mathrm{e}$ and $4 \mathrm{f}$ shows the surface patterns formed with the copper tubes of different inner diameters. The height of the copper tubes were $6 \mathrm{~mm}$. It is evident that the surface patterns gradually evolve from the concentric rings of linked-beads to the wave-like rings as the inner diameter increases, and the average wavelength increases with the increase of the inner diameter. The larger the inner diameter of the copper tube, the more is the space available for the evaporation of the acetone in the polymer solution. There is a lower gradient of the evaporation rate in a copper tube of larger diameter than that in a copper tube of smaller diameter, and concentric rings with more wave-like rings are formed. The uneven evaporation over the surface of the polymer solution film can introduce local perturbation in the vicinity of the CL and the gradient of surface tension, which cause local instabilities and the formation of concentric rings with linked beads.

From the optical images, one can measure the wavelength of the concentric rings as a function of the distance to the inner surface of the copper tube. Figure 5 shows the variation of the wavelength of concentric rings with the distance to the inner surface of copper tubes of different inner diameters and heights. The substrate temperature was $50^{\circ} \mathrm{C}$, and the concentration of PMMA was $0.125 \mathrm{wt} \%$. For each individual copper tube, the wavelength of the corresponding concentric rings is relatively independent of the distance to the inner surface of the copper tube in accordance with the above observation, while it is in contrast to the gradient-concentric rings formed by using a copper ring [28] as the template or other widely-used templates (i.e. 'sphere-on-flat') [40]. The use of a copper tube as a template to confine the evaporation of the volatile solvent in a polymer solution provides a possible approach to form concentric rings with spatial-independent wavelength.

Figure $5 d$ shows the variation of the wavelength of concentric rings with the height of copper tube for different inner diameters. For the copper tubes of the same inner diameter, the wavelength decreases with 


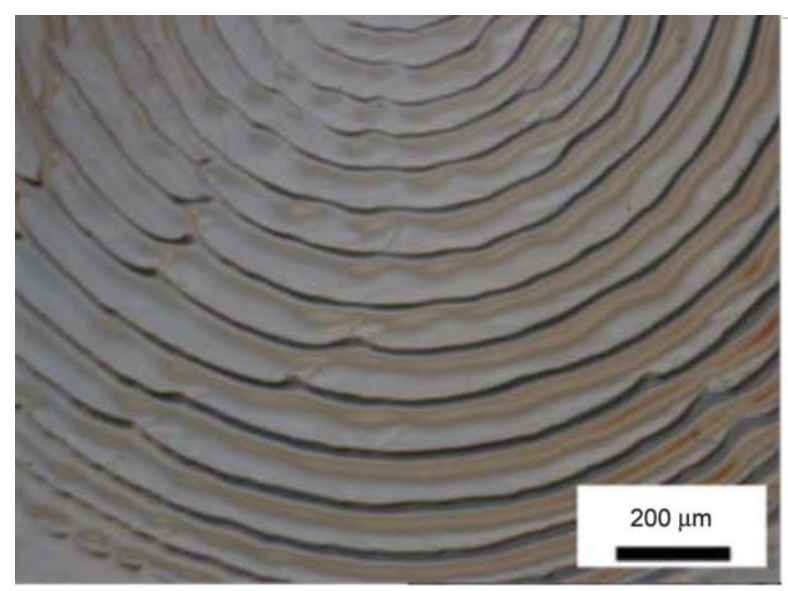

a)

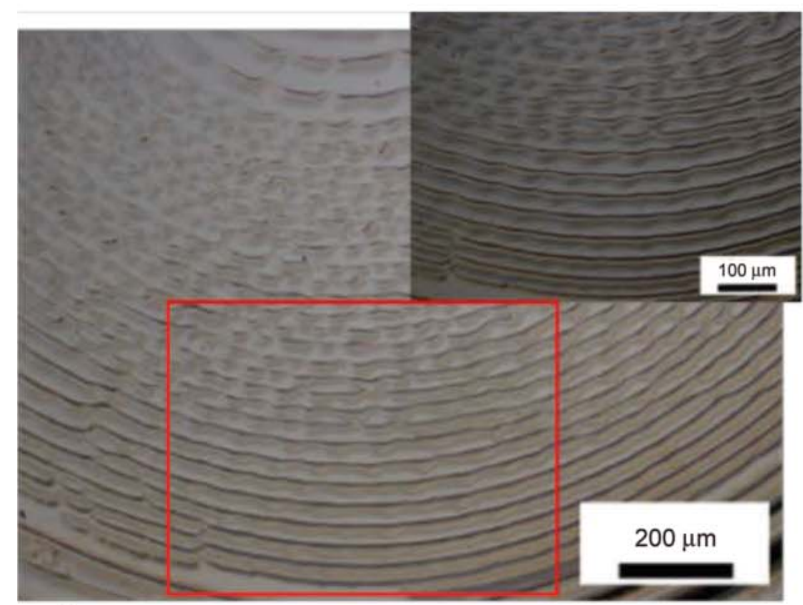

c)

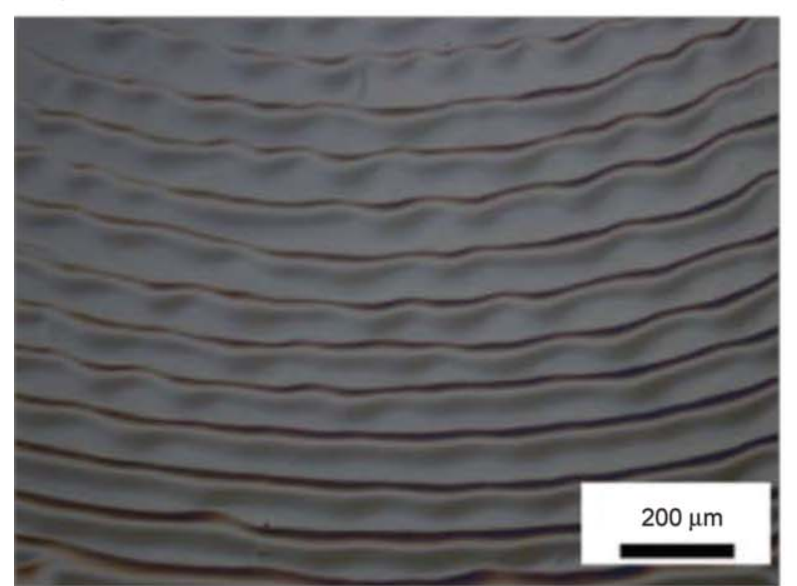

e)

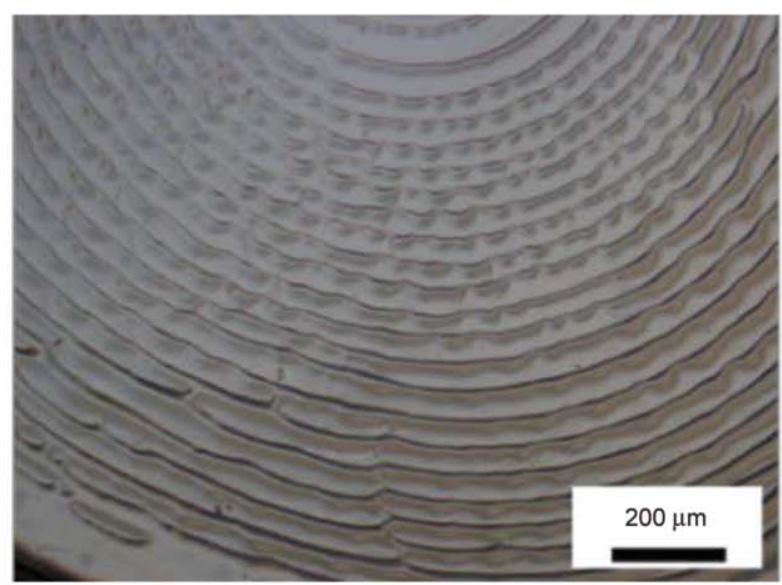

b)

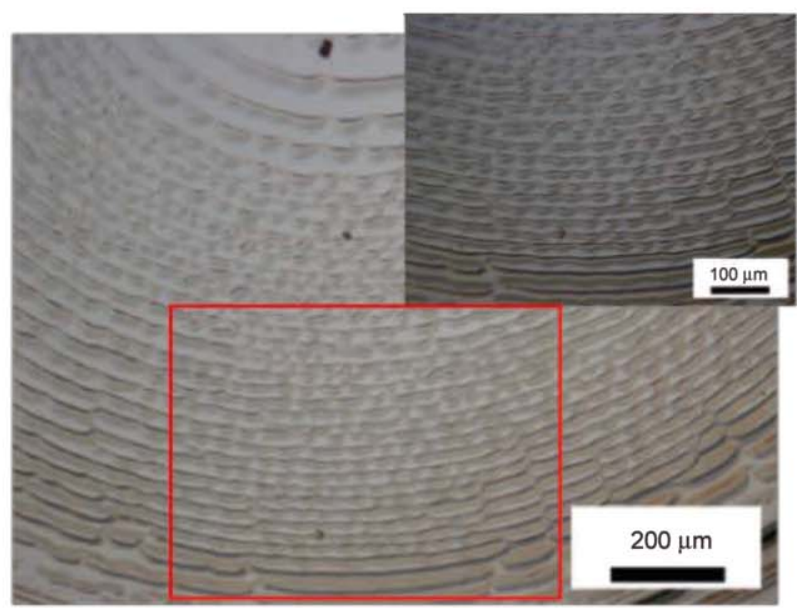

d)

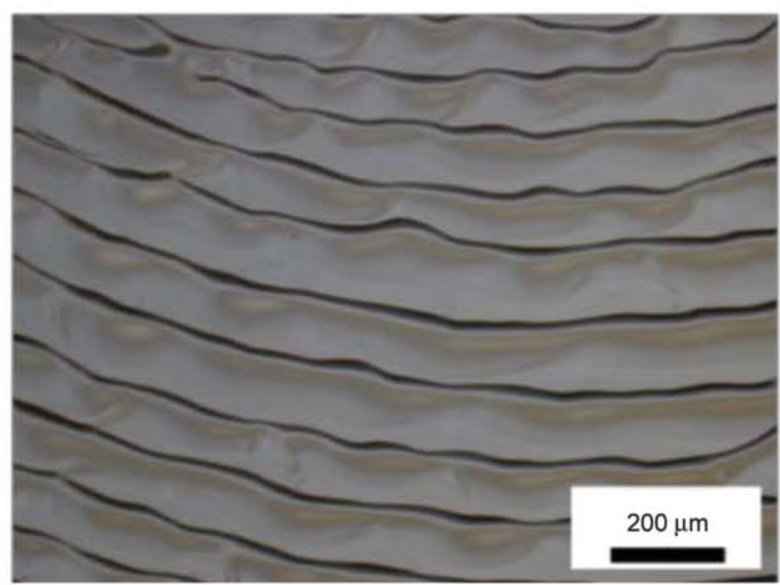

f)

Figure 4. Optical images of the concentric-ring patterns formed via the evaporation of a droplet of the acetone solution with $0.1 \mathrm{wt} \%$ PMMA within copper tubes of different dimensions (substrate temperature: $50^{\circ} \mathrm{C}$ ): (a) $2.5 \mathrm{~mm}$ in inner diameter and $1 \mathrm{~mm}$ in height, (b) $2.5 \mathrm{~mm}$ in inner diameter and $3 \mathrm{~mm}$ in height, (c) $2.5 \mathrm{~mm}$ in inner diameter and $6 \mathrm{~mm}$ in height (inset: enlarged view of the patterns in the red box), (d) $2.5 \mathrm{~mm}$ in inner diameter and $9 \mathrm{~mm}$ in height (inset: enlarged view of the patterns in the red box), (e) $4.5 \mathrm{~mm}$ in inner diameter and $6 \mathrm{~mm}$ in height, and (f) $6.5 \mathrm{~mm}$ in inner diameter and $6 \mathrm{~mm}$ in height.

the increase of the tube height, suggesting that the tube height plays an important role in controlling the evaporation of the acetone in the polymer solution, which is confined in the copper tube, and the geometric characteristics of the surface patterns.

\subsection{Effect of substrate temperature}

Figure $6 \mathrm{a}-\mathrm{d}$ shows optical images of the surface patterns formed at a substrate temperature of $30^{\circ} \mathrm{C}$ by using templates of copper tubes of $2.5 \mathrm{~mm}$ in inner diameter and different tube heights. In contrast to the 

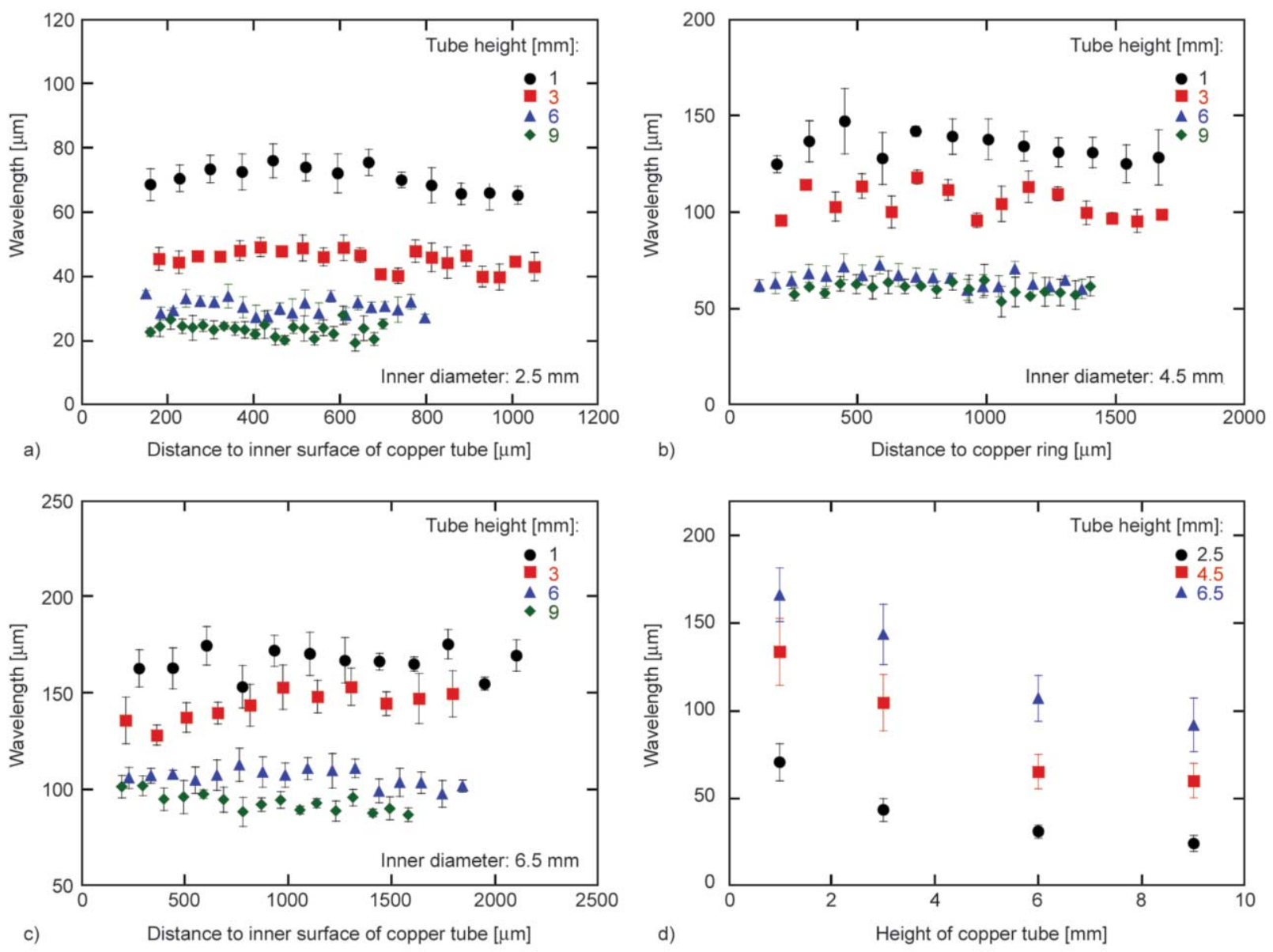

Figure 5. Variation of the wavelength of concentric rings with the distance to the inner surface of copper tubes of different inner diameters (substrate temperature: $50^{\circ} \mathrm{C}$, concentration of PMMA: $0.125 \mathrm{wt} \%$ ): (a) $2.5 \mathrm{~mm}$, (b) $4.5 \mathrm{~mm}$, (c) $6.5 \mathrm{~mm}$, and (d) variation of the wavelength of concentric rings with the height of copper tube for different inner diameters.

concentric rings formed at $50^{\circ} \mathrm{C}$, which cover almost the entire surface of the glass slide within the copper tubes of all the dimensions tested, most concentric rings formed by using the copper tubes of the same dimensions only cover small portion of the surface of the glass slide within the copper tube. There is a large, featureless zone for most surface patterns. Note that the concentric rings formed by using the copper tubes of $1 \mathrm{~mm}$ in height cover almost the entire surface of the glass slide within the copper tubes, similar to the surface patterns formed at $50^{\circ} \mathrm{C}$. Note that the formation and size of the featureless zone is determined by the first 'stick' state of the CL. For a small evaporation rate, the CL tends to move smoothly at a low speed, and takes relatively long time to reach the first 'stick' state; the viscosity of the polymer solution increases slowly. For a high evaporation rate, the viscosity of the polymer solution increases rapidly, leading to the increase of the resistance to the CL motion. The CL can reach the first 'stick' state restively quickly to form the first concentric ring.
That is to say, the size of the featureless zone is dependent on the evaporation rate. Increasing the height of copper tube limits the evaporation of the acetone in the polymer solution film, and it is difficult for the solvent vapor above the polymer solution film to diffuse out of the copper tube in a short time. This trend suggests that using a copper tube of a larger height can decrease the evaporation rate, and lead to the formation of a featureless zone of a larger size. Figure $6 \mathrm{~b}, 6 \mathrm{e}$ and $6 \mathrm{f}$ shows optical images of the surface patterns formed by using a copper tube of $2.5 \mathrm{~mm}$ in inner diameter and $6 \mathrm{~mm}$ in height at different substrate temperatures of 30,50 , and $20^{\circ} \mathrm{C}$. The surface of the polymer film after complete evaporation of the acetone in the polymer solution exhibits wrinkling-like topology, partial concentric rings, and concentric rings over the surfaces of the glass slides for the substrate temperatures of 20,30 , and $50^{\circ} \mathrm{C}$, respectively. Such a result reveals the importance of the substrate temperature in controlling the formation of surface structures induced by the evaporation 


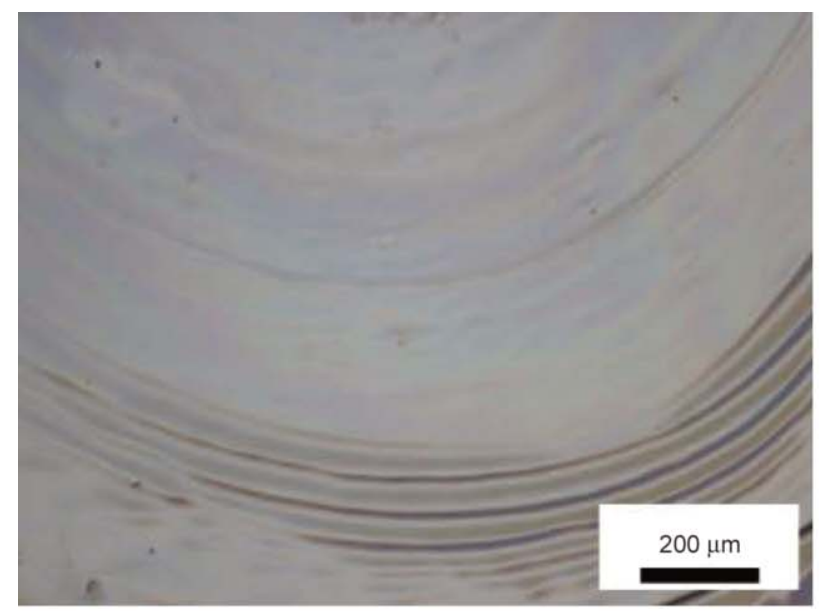

a)

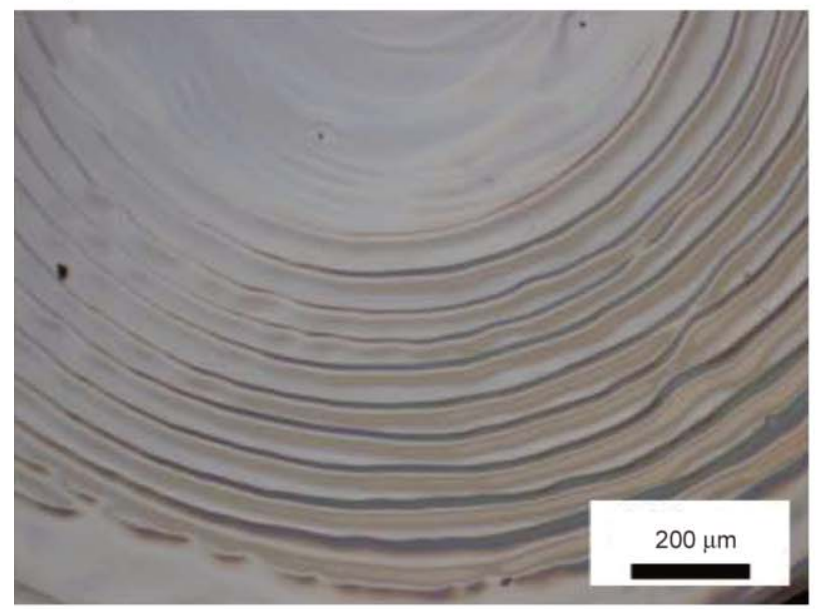

c)

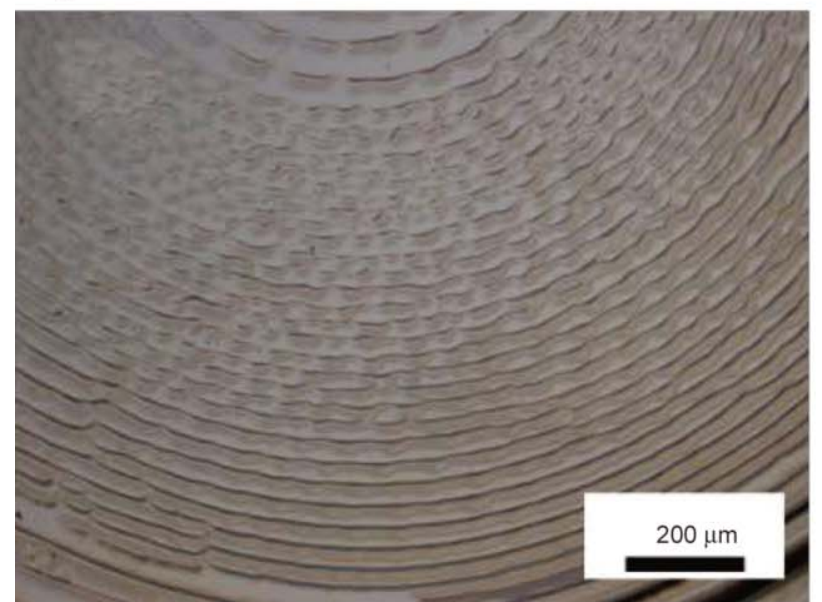

e)

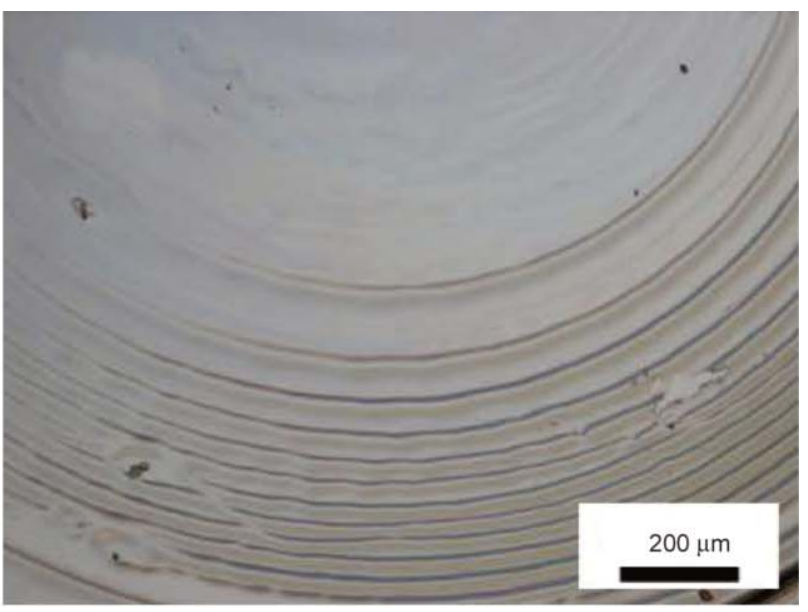

b)

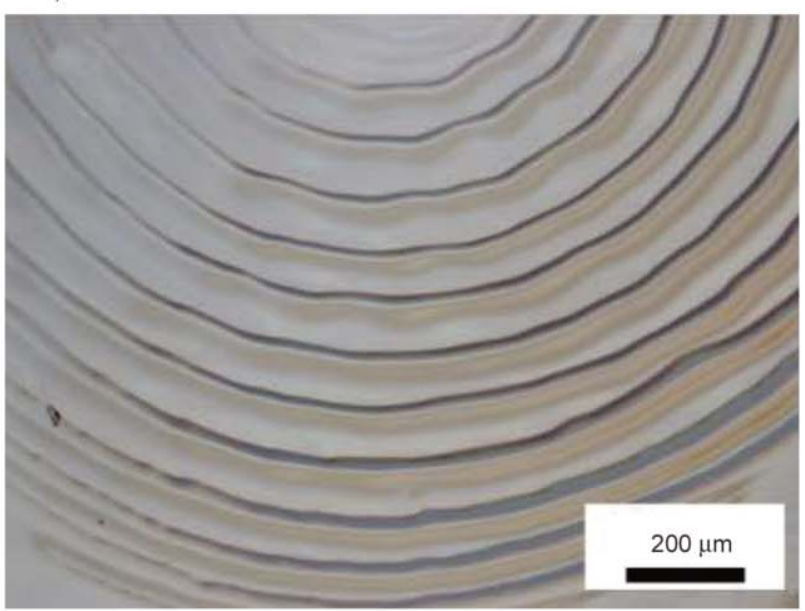

d)

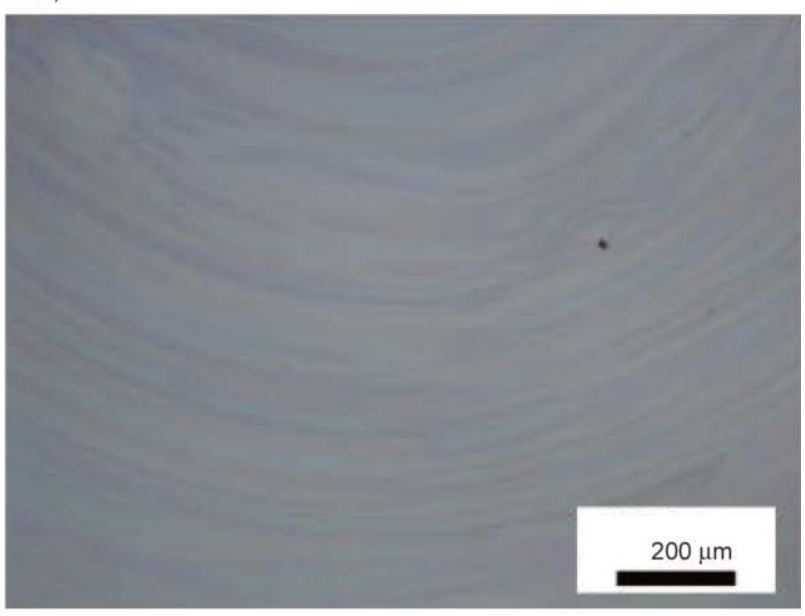

f)

Figure 6. (a-d) Optical images of the surface patterns formed by using copper tubes of $2.5 \mathrm{~mm}$ in inner diameter for different tube heights (substrate temperature: $30^{\circ} \mathrm{C}$ ): (a) $9 \mathrm{~mm}$, (b) $6 \mathrm{~mm}$, (c) $3 \mathrm{~mm}$, and (d) $1 \mathrm{~mm}$; and (e-f) optical images of the surface patterns formed by using a copper tube of $2.5 \mathrm{~mm}$ in inner diameter and $6 \mathrm{~mm}$ in height at different substrate temperatures: (e) $50^{\circ} \mathrm{C}$, and (f) $20^{\circ} \mathrm{C}$.

of a polymer solution. Also, the distortions of the concentric rings associated with the evaporation-induced instabilities are more severe for the patterns formed at $50^{\circ} \mathrm{C}$ than that at $30^{\circ} \mathrm{C}$, in accordance with the conclusion given by De Gennes [35] that it is easy to induce instability at a high vapor pressure. It is known that both the evaporation rate of solvent and the viscosity of the polymer solution are 
temperature-dependent. Increasing temperature leads to the increase of the evaporation rate of solvent and the decrease of the viscosity of the polymer solution, while the fast evaporation of solvent causes the increase of the viscosity of the polymer solution. For a polymer solution with volatile solvent, increasing temperature likely causes the decrease of the viscosity of the polymer solution first and the increase of the viscosity of the polymer solution due to the evaporation of the solvent after heating for long time. Generally, the formation of the first CL is dependent on the evaporation of the solvent. Slow evaporation of the solvent can inhibit the formation of the first $\mathrm{CL}$, which limits the formation of surface structures. Also, the speed of the CL motion decreases with the increase of the viscosity of the polymer solution. At a low temperature, the polymer solution has a large viscosity, which makes it difficult to have the stickslip motion of the CL, as supported by Figure $6 f$. Figure 7 depicts variation of the wavelengths of the concentric rings formed at different substrate temperatures with the height of copper tube for three

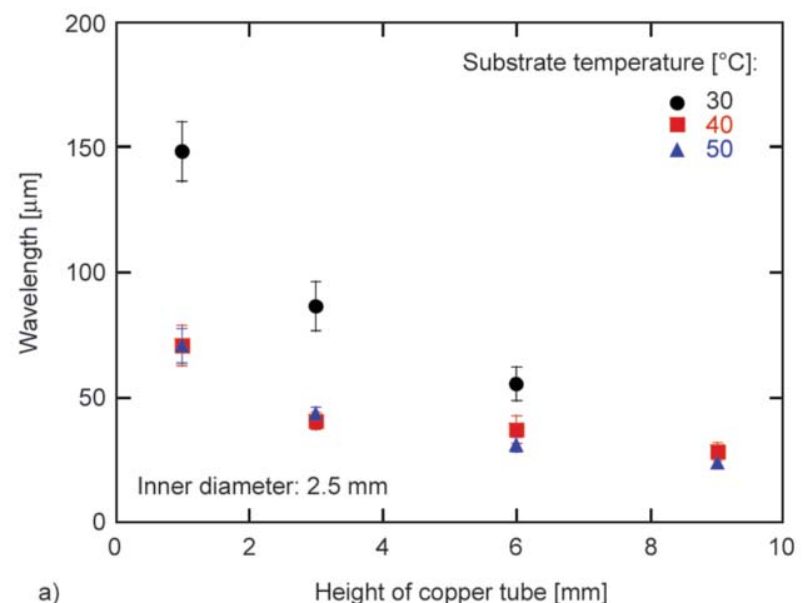

inner diameters of $2.5,4.5$, and $6.5 \mathrm{~mm}$. The wavelength decreases with the increase of the height of the copper tube for all the three substrate temperatures. Such behavior indirectly reveals the effect of the evaporation of the solvent in the polymer solution on the characteristics of the concentric rings. For copper tubes of a larger height, it takes a longer time for the solvent vapor to diffuse out of the copper tubes. This trend reduces the non-uniformity of the evaporation of the solvent over the surface of the polymer solution and the gradient of the evaporation rate along radial direction from the center to the inner surface of the copper tubes, resulting in smaller wavelength.

Figure 8 shows typical surface patterns formed via the evaporation of a droplet of the PMMA solution confined by copper tubes of $6.5 \mathrm{~mm}$ in inner diameter at a substrate temperature of $10^{\circ} \mathrm{C}$. Instead of forming concentric rings for the substrate temperature larger than or equal to $30^{\circ} \mathrm{C}$, surface patterns consisting of 'hole-network' were formed. The formation of these surface patterns can be attributed to the

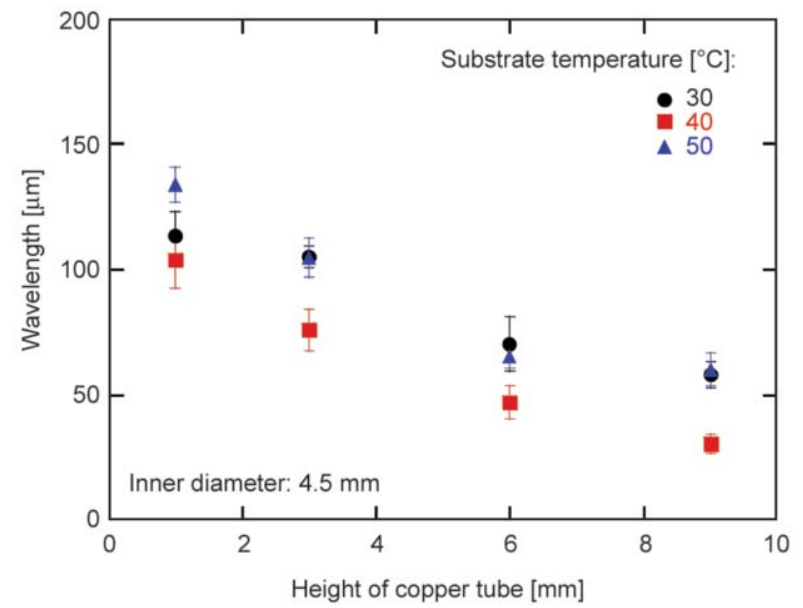

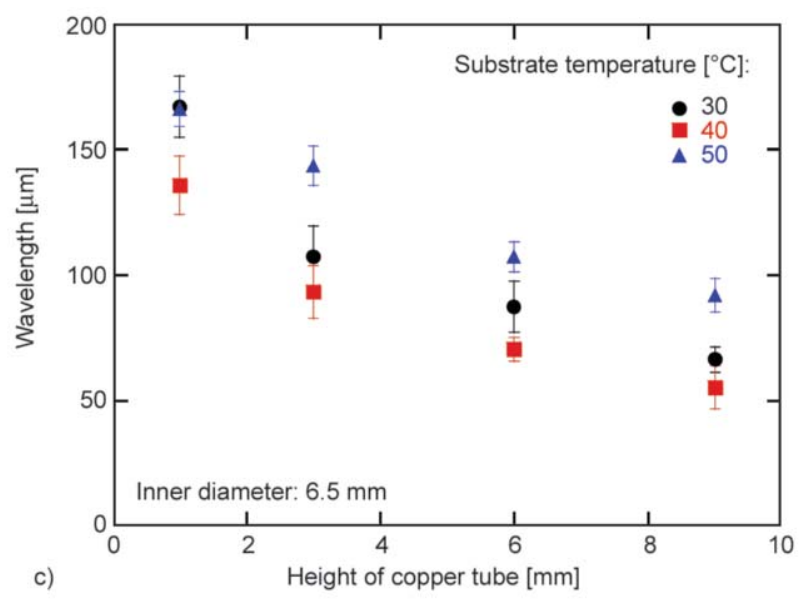

Figure 7. Variation of the wavelengths of the concentric rings formed at different substrate temperatures with the height of copper tube for different inner diameters: (a) $2.5 \mathrm{~mm}$, (b) $4.5 \mathrm{~mm}$, and (c) $6.5 \mathrm{~mm}$. 

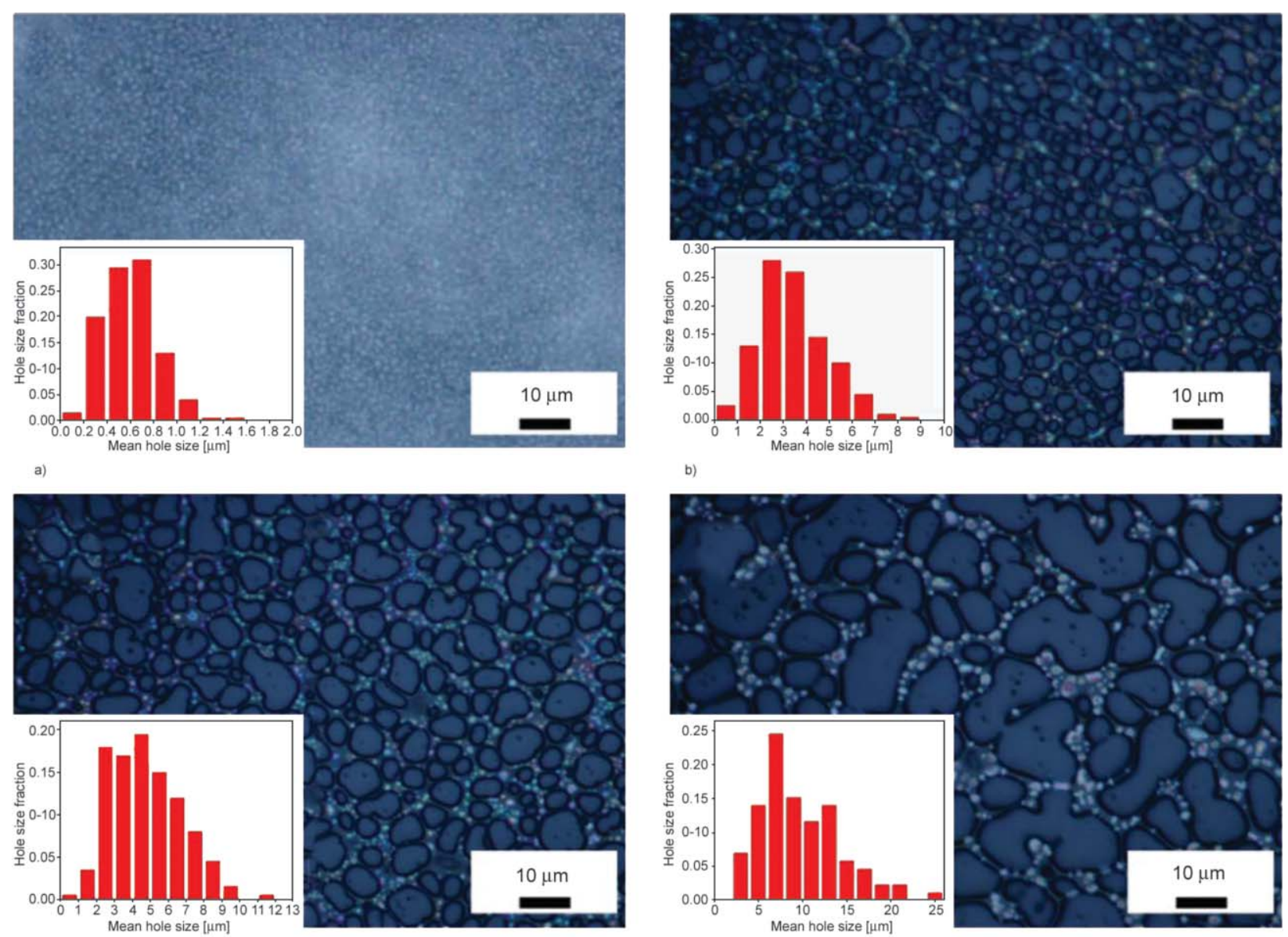

$$
\text { b) }
$$
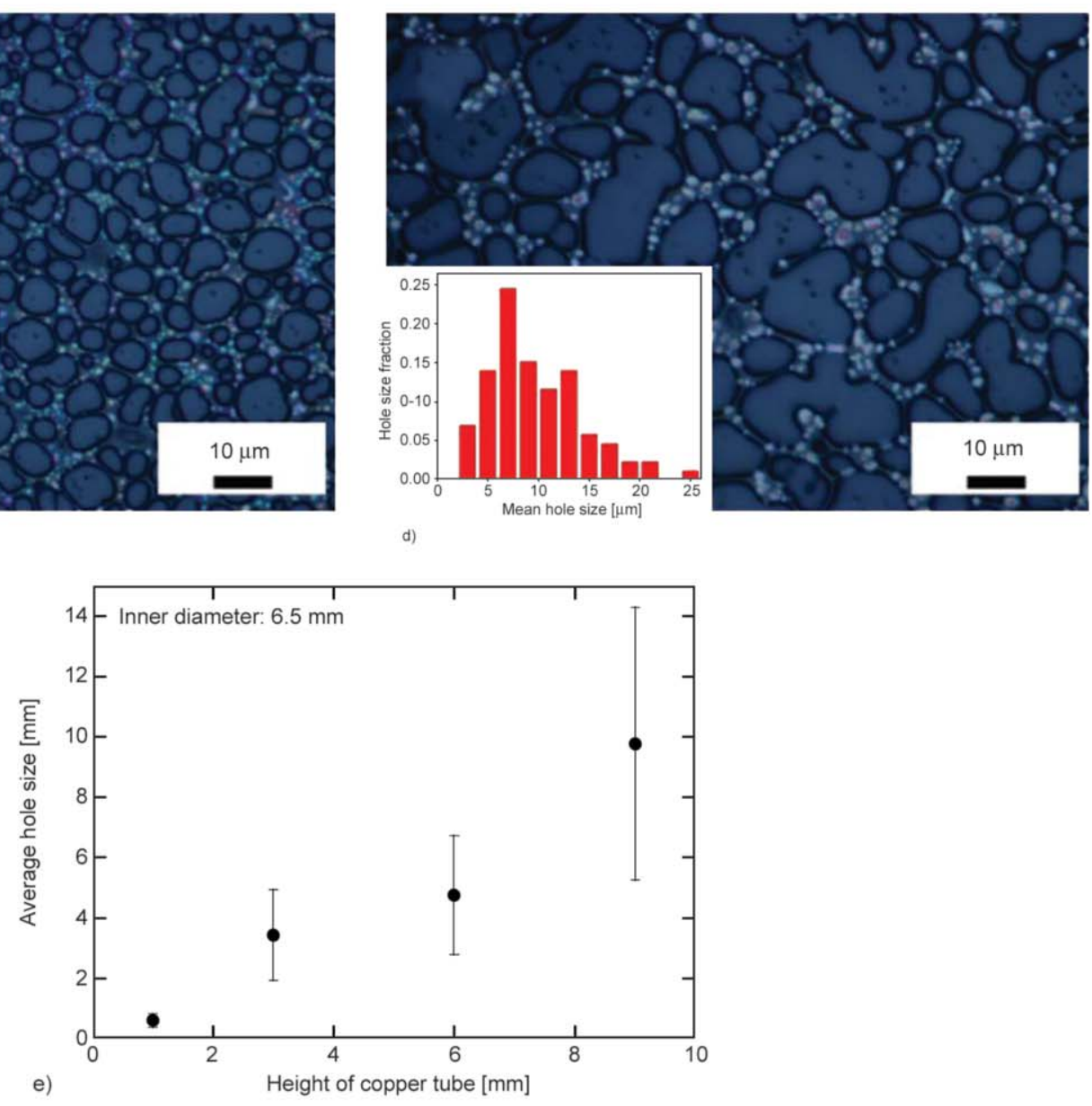

Figure 8. (a-d) Optical images of surface patterns formed with copper tubes for different tube heights: (a) $1 \mathrm{~mm}$, (b) $3 \mathrm{~mm}$, (c) $6 \mathrm{~mm}$, and (d) $9 \mathrm{~mm}$ (inset: distribution of hole sizes); (e) variation of the average hole size with the height of copper tube (inner diameter: $6.5 \mathrm{~mm}$, substrate temperature: $10^{\circ} \mathrm{C}$ ).

'Breath Figure' phenomenon [41], which usually occurs during the condensation of water droplets onto the surface of a polymer solution with a volatile solvent in a high humidity environment. The evaporation of the volatile solvent in the polymer solution causes the decrease of local temperature, leading to the formation of water micro-droplets and the penetration of the water micro-droplets onto the layer of the polymer solution. The micro-droplets grow through coalescence, and the droplets in the layer of the polymer solution serve as a template for the construction of surface patterns on the surface of the polymer film after both the droplets and the solvent evaporate completely.

For the PMMA solution on a glass substrate of $10^{\circ} \mathrm{C}$, the evaporation of acetone further reduces the 
temperature of the layer of the PMMA solution, which increases the temperature difference between ambient temperature and the temperature on the surface of the polymer layer and leads the condensation of water vapor even at a humidity of $\sim 30 \%$. The condensation of water vapor results in the formation of micro-droplets, which drop onto the polymer solution and induce the 'Breath Figure' phenomenon as observed in Figure 8.

From Figure 8, one can note that the surface structures consist of holes of irregular shapes in contrast to circular holes and/or well-ordered hexagonal holes formed in a system of two immiscible liquids [29]. Such behavior is likely due to that water can completely mix with acetone, which locally forms a homogeneous and isotropic liquid with two different evaporation rates. The average size of the irregular holes is represented by the diameter of an equivalent circle of the same area as the hole, which was analyzed by Image-pro plus software. The average size of the holes increases with the increase of the height of the copper tube, as shown in Figure 8e. With the increase of the tube height, the evaporation rate of acetone decreases, and there is more time for the coalescence of water droplets, leading to the template with large water droplets and the formation of surface patterns of large holes. There is a broad distribution of the hole sizes (see the insets of Figure 8ad). It is worth mentioning that further suppressing the evaporation rate via the increase of the tube height and/or the decrease of the substrate temperature cannot create 'Breath Figure' patterns due to that the formation of a homogeneous and isotropic PMMA solution consisting of water and acetone.

Table 1 summarize the types of the surface patterns formed via the evaporation of the droplets of the acetone solution of $0.1 \mathrm{wt} \%$ PMMA on the surfaces of glass slides using the copper tubes as the templates at different substrate temperatures. It is evident that the substrate temperature plays a major role in determining the types of the surface patterns, while the dimensions of the copper tubes also have an effect on the formation of the surface patterns through the control of the evaporation of acetone. In general, the surface patterns vary from concentric rings of wavelike shape, concentric rings of linked-beads, wrinkling, 'Breath Figure' patterns to structure-less surface, and are dependent on the CL motion, instability induced by local disturbances, and the template. For the same geometrical dimensions of copper tubes, increasing the evaporation rate of acetone leads to the increase of the wavelength of the concentric rings. However, increasing the substrate temperature contributes to the increase of the evaporation rate and the change of the viscosity of the polymer solution. There exists an interaction between the increase of the evaporation rate and the change of the viscosity of the polymer solution, which determines the evolution of surface patterns. Increasing the substrate temperature does not lead to a monotonic increase

Table 1. Summary of the types and wavelength of the surface patterns formed with different tube dimensions and substrate temperatures.

\begin{tabular}{|c|c|c|c|c|c|c|c|}
\hline \multicolumn{2}{|c|}{ Tube dimensions } & \multicolumn{6}{|c|}{$\begin{array}{l}\text { Substrate temperature } \\
\qquad\left[{ }^{\circ} \mathbf{C}\right]\end{array}$} \\
\hline $\begin{array}{l}\text { Inner diameter } \\
{[\mathrm{mm}]}\end{array}$ & $\begin{array}{l}\text { Height } \\
{[\mathrm{mm}]}\end{array}$ & $\mathbf{0}$ & 10 & 20 & 30 & 40 & 50 \\
\hline \multirow{4}{*}{2.5} & 1 & bf & bf & ir & $\operatorname{cr}(148)$ & $\operatorname{cr}(71)$ & $\operatorname{cr}(70)$ \\
\hline & 3 & no & bf & ir+wk & cr+wk (86) & $\mathrm{cr}+\mathrm{lb}(41)$ & $\mathrm{cr}+\mathrm{lb}(43)$ \\
\hline & 6 & no & wk & wk & cr+wk (56) & $\mathrm{cr}+\mathrm{lb}(37)$ & $\mathrm{cr}+\mathrm{lb}(31)$ \\
\hline & 9 & no & wk & wk & $\mathrm{cr}+\mathrm{wk}$ & $\mathrm{lb}(28)$ & $\mathrm{lb}(24)$ \\
\hline \multirow{4}{*}{4.5} & 1 & bf & bf & ir+wk & cr (113) & $\operatorname{cr}(103)$ & $\operatorname{cr}(133)$ \\
\hline & 3 & bf & bf & ir+wk & $\mathrm{cr}+\mathrm{lb}(104)$ & $\operatorname{cr}(76)$ & $\operatorname{cr}(104)$ \\
\hline & 6 & bf & bf & wk & $\mathrm{cr}+\mathrm{lb}(70)$ & $\mathrm{cr}+\mathrm{lb}(47)$ & $\mathrm{cr}+\mathrm{lb}(65)$ \\
\hline & 9 & no & bf & wk & cr+wk (58) & $\mathrm{cr}+\mathrm{lb}(30)$ & $\mathrm{cr}+\mathrm{lb}(60)$ \\
\hline \multirow{4}{*}{6.5} & 1 & bf & bf & ir+wk & $\operatorname{cr}(168)$ & $\operatorname{cr}(135)$ & cr (166) \\
\hline & 3 & bf & bf & wk & $\mathrm{cr}+\mathrm{lb}(107)$ & $\operatorname{cr}(93)$ & $\operatorname{cr}(143)$ \\
\hline & 6 & bf & bf & wk & $\mathrm{cr}+\mathrm{lb}(87)$ & $\operatorname{cr}(70)$ & cr (107) \\
\hline & 9 & bf & bf & wk & $\mathrm{cr}+\mathrm{lb}(66)$ & $\mathrm{cr}+\mathrm{lb}(55)$ & $\operatorname{cr}(91)$ \\
\hline
\end{tabular}

Pattern type: cr: concentric rings, lb: linked beads, wk: wrinkling, bf: 'Breath Figure' pattern, ir: irregular ring, no: no typical pattern. Two types linked by '+' means the combination of the two types. The wavelengths (in the unit of $\mu \mathrm{m}$ ) of the concentric rings with and/or without linked beads are listed in the bracket. 
of the wavelength due to the variation of local viscosity with temperature and evaporation time. The concentric rings formed at the substrate temperature of $40^{\circ} \mathrm{C}$ have the smallest wavelength due to the coupling between the evaporation and the change of local viscosity for most copper tubes used.

It is believed that this technique or similar procedure has potential to produce well-ordered structures of submicro- and nano-scales, even though the patterns constructed in this work are mainly at the microscale. To reduce the characteristic dimension of the periodic patterns to submicro- and nano-scales, one needs to carefully control the experimental conditions, including the film thickness, viscosity and the radius of the copper tube, to significantly reduce the traveling distance of the CL during the slip state. For the 'Breath Figure' patterns, the key is to form stable nano-template (i.e. nano droplets) by controlling the sizes of water droplets during condensation.

There are several important factors significantly controlling the geometry and dimensions of the formed patterns, including the geometrical dimensions of template, temperature, properties of polymer, solvent, solution concentration, and surface properties of substrate. With reasonable combination of materials and experimental conditions, it is possible to achieve nanostructuration via the 'evaporation-induced' patterning technique.

\section{Conclusions}

In summary, copper tubes have been used as the templates to construct surface patterns on the surfaces of the polymer films, which are confined by the copper tubes. The function of the copper tubes is to limit the evaporation of the volatile solvent in the polymer solution. The parameters affecting the evaporation of the volatile solvent include the height and inner diameter of the copper tube and the substrate temperature, and their effects on the geometric characteristics of the surface patterns have been systematically studied. The surface patterns vary from 'Breath Figure' patterns formed at low temperatures to concentric rings of wave-like shape and/or linked-beads formed at high temperatures, which are associated with the condensation of water micro-droplets and the instability due to local disturbance, respectively. The substrate temperature plays a major role in controlling the formation of the surface patterns, since both the evaporation behavior of the volatile solvent and the viscosity of the polymer solution are temperature-dependent. In addition, the evaporation of the volatile solvent can cause the change of the viscosity of the polymer solution, leading to the coupling effect.

In contrast to the gradient-concentric rings reported in literature, the wavelength of the concentric rings formed is relatively independent of the distance to the inner surface of the copper tube. The longer the copper tube of the same inner diameter, the smaller is the wavelength. By varying the substrate temperature and the dimensions of the copper tube, one can construct concentric rings with the wavelength in a range of 20 to $170 \mu \mathrm{m}$.

The technique developed in this work opens a new route to fabricate surface patterns with tunable topologies by simply controlling the evaporation of volatile solvent in polymer solutions through the substrate temperature and the dimensions of the copper tube. It also presents challenge to understand the effect of geometrical dimensions of solid objects on the motion of the three-phase contact line for producing surface textures of micro- and nanoscales. It needs further study to understand the effects of other factors, such as the surface properties of template, solvent, molecular weight, for the optimization of this technique.

\section{Acknowledgements}

SW is grateful for the financial support from the Doctorial startup foundation of Liaoning (No. 20170520392) and General project of Liaoning Education Department (No. L2017LQN014)

\section{References}

[1] Khang D-Y., Jiang H., Huang Y., Rogers J. A.: A stretchable form of single-crystal silicon for high-performance electronics on rubber substrates. Science, 311, 208-212 (2006). https://doi.org/10.1126/science.1121401

[2] Kolaric B., Vandeparre H., Desprez S., Vallée R. A., Damman P.: In situ tuning the optical properties of a cavity by wrinkling. Applied Physics Letters, 96, 043119/1-043119/3 (2010).

https://doi.org/10.1063/1.3298740

[3] Kikuchi A., Okano T.: Nanostructured designs of biomedical materials: Applications of cell sheet engineering to functional regenerative tissues and organs. Journal of Controlled Release, 101, 69-84 (2005).

https://doi.org/10.1016/j.jconrel.2004.08.026

[4] Langer R., Tirrell D. A.: Designing materials for biology and medicine. Nature, 428, 487-492 (2004). https://doi.org/10.1038/nature02388 
[5] Fukuhira Y., Kitazono E., Hayashi T., Kaneko H., Tanaka M., Shimomura M., Sumi Y.: Biodegradable honeycomb-patterned film composed of poly(lactic acid) and dioleoylphosphatidylethanolamine. Biomaterials, 27, 1797-1802 (2006).

https://doi.org/10.1016/j.biomaterials.2005.10.019

[6] Chmiola J., Largeot C., Taberna P-L., Simon P., Gogotsi Y.: Monolithic carbide-derived carbon films for microsupercapacitors. Science, 328, 480-483 (2010). https://doi.org/10.1126/science.1184126

[7] Kim S-K., Koo H-J., Lee A., Braun P. V.: Selective wetting-induced micro-electrode patterning for flexible micro-supercapacitors. Advanced Materials, 26, 51085112 (2014).

https://doi.org/10.1002/adma.201401525

[8] Cavallini M., Gomez-Segura J., Albonetti C., RuizMolina D., Veciana J., Biscarini F.: Ordered patterning of nanometric rings of single molecule magnets on polymers by lithographic control of demixing. The Journal of Physical Chemistry B, 110, 11607-11610 (2006). https://doi.org/10.1021/jp061231g

[9] Chan E. P., Smith E. J., Hayward R. C., Crosby A. J.: Surface wrinkles for smart adhesion. Advanced Materials, 20, 711-716 (2008).

https://doi.org/10.1002/adma.200701530

[10] Zhou W., Min G., Zhang J., Liu Y., Wang J., Zhang Y., Sun F.: Nanoimprint lithography: A processing technique for nanofabrication advancement. Nano-Micro Letters, 3, 135-140 (2011).

https://doi.org/10.3786/nml.v3i2.p135-140

[11] Zhang Z., Zhang X., Xin Z., Deng M., Wen Y., Song Y.: Controlled inkjetting of a conductive pattern of silver nanoparticles based on the coffee ring effect. Advanced Materials, 25, 6714-6718 (2013). https://doi.org/10.1002/adma.201303278

[12] Gao W., Singh N., Song L., Liu Z., Reddy A. L. M., Ci L., Vajtai R., Zhang Q., Wei B., Ajayan P. M.: Direct laser writing of micro-supercapacitors on hydrated graphite oxide films. Nature Nanotechnology, 6, 496500 (2011).

https://doi.org/10.1038/nnano.2011.110

[13] Pease L. F., Deshpande P., Wang Y., Russel W. B., Chou S. Y.: Self-formation of sub-60-nm half-pitch gratings with large areas through fracturing. Nature Nanotechnology, 2, 545-548 (2007).

https://doi.org/10.1038/nnano.2007.264

[14] Deegan R. D., Bakajin O., Dupont T. F., Huber G., Nagel S. R., Witten T. A.: Capillary flow as the cause of ring stains from dried liquid drops. Nature, 389, 827829 (1997).

https://doi.org/10.1038/39827

[15] Deegan R. D.: Pattern formation in drying drops. Physical Review E, 61, 475-485 (2000).

https://doi.org/10.1103/PhysRevE.61.475
[16] Deegan R. D., Bakajin O., Dupont T. F., Huber G., Nagel S. R., Witten T. A.: Contact line deposits in an evaporating drop. Physical Review E, 62, 756-765 (2000). https://doi.org/10.1103/PhysRevE.62.756

[17] Bae D. G., Jeong J-E., Kang S. H., Byun M., Han DW., Lin Z., Woo H. Y., Hong S. W.: A nonconventional approach to patterned nanoarrays of DNA strands for template-assisted assembly of polyfluorene nanowires. Small, 12, 4254-4263 (2016). https://doi.org/10.1002/smll.201601346

[18] Byun M., Hong S. W., Qiu F., Zou Q., Lin Z.: Evaporative organization of hierarchically structured polymer blend rings. Macromolecules, 41, 9312-9317 (2008). https://doi.org/10.1021/ma801864n

[19] Byun M., Hong S. W., Zhu L., Lin Z.: Self-assembling semicrystalline polymer into highly ordered, microscopic concentric rings by evaporation. Langmuir, 24, 3525-3531 (2008). https://doi.org/10.1021/la703270c

[20] Han W., Byun M., Li B., Pang X., Lin Z.: A simple route to hierarchically assembled micelles and inorganic nanoparticles. Angewandte Chemie International Edition, 51, 12756-12760 (2012).

https://doi.org/10.1002/anie.201207902

[21] Han W., He M., Byun M., Li B., Lin Z.: Large-scale hierarchically structured conjugated polymer assemblies with enhanced electrical conductivity. Angewandte Chemie International Edition, 52, 2564-2568 (2013). https://doi.org/10.1002/anie.201209632

[22] Xiao L., Wei J., Gao Y., Yang D., Li H.: Formation of gradient multiwalled carbon nanotube stripe patterns by using evaporation-induced self-assembly. ACS Applied Materials and Interfaces, 4, 3811-3817 (2012). https://doi.org/10.1021/am300936a

[23] Park W. K., Kim T., Kim H., Kim Y., Tung T. T., Lin Z., Jang A-R., Shin H. S., Han J. H., Yoon D. H., Yang W. S.: Large-scale patterning by the roll-based evaporation-induced self-assembly. Journal of Materials Chemistry, 22, 22844-22847 (2012). https://doi.org/10.1039/C2JM34212J

[24] Li B., Zhang C., Jiang B., Han W., Lin Z.: Flow-enabled self-assembly of large-scale aligned nanowires. Angewandte Chemie International Edition, 54, 42504254 (2015). https://doi.org/10.1002/anie.201412388

[25] Lin Y., Su Z., Xiao G., Balizan E., Kaur G., Niu Z., Wang Q.: Self-assembly of virus particles on flat surfaces via controlled evaporation. Langmuir, 27, 13981402 (2010). https://doi.org/10.1021/la103917x

[26] Byun M., Han W., Li B., Xin X., Lin Z.: An unconventional route to hierarchically ordered block copolymers on a gradient patterned surface through controlled evaporative self-assembly. Angewandte Chemie International Edition, 52, 1122-1127 (2013). https://doi.org/10.1002/anie.201208421 
[27] Poulard C., Damman P.: Control of spreading and drying of a polymer solution from Marangoni flows. Europhysics Letters, 80, 64001/1-64001/5 (2007). https://doi.org/10.1209/0295-5075/80/64001

[28] Sun W., Yang F.: Fabrication of asymmetric-gradientconcentric ring patterns via evaporation of droplets of PMMA solution at different substrate temperatures. RSC Advances, 5, 29850-29858 (2015). https://doi.org/10.1039/C5RA01659B

[29] Sun W., Yang F.: Cooling-induced formation of honeycomb patterns on pre-cast PMMA films at low temperatures. RSC Advances, 5, 60496-60505 (2015). https://doi.org/10.1039/C5RA09579D

[30] Yu Y., Ma Y.: Breath figure fabrication of honeycomb films with small molecules through hydrogen bond mediated self-assembly. Soft Matter, 7, 884-886 (2011). https://doi.org/10.1039/C0SM01346C

[31] Kelly-Zion P. L., Pursell C. J., Booth R. S., Van Tilburg A. N.: Evaporation rates of pure hydrocarbon liquids under the influences of natural convection and diffusion. International Journal of Heat and Mass Transfer, 52, 3305-3313 (2009).

https://doi.org/10.1016/j.ijheatmasstransfer.2009.01.015

[32] Sun W., Yang F.: Self-organization of unconventional gradient concentric rings on precast PMMA films. The Journal of Physical Chemistry C, 118, 10177-10182 (2014). https://doi.org/10.1021/jp502304m

[33] Sun W., Yang F.: Evaporation-induced formation of self-organized gradient concentric rings on sub-micron pre-cast PMMA films. Soft Matter, 10, 4451-4457 (2014). https://doi.org/10.1039/c4sm00245h

[34] Mitov Z., Kumacheva E.: Convection-induced patterns in phase-separating polymeric fluids. Physical Review Letters, 81, 3427/1-3427/4 (1998).

https://doi.org/10.1103/PhysRevLett.81.3427
[35] De Gennes P. G.: Instabilities during the evaporation of a film: Non-glassy polymer + volatile solvent. The European Physical Journal E, 6, 421-424 (2001). https://doi.org/10.1007/s10189-001-8055-3

[36] De Gennes P. G.: Solvent evaporation of spin cast films: 'Crust' effects. The European Physical Journal E, 7, 3134 (2002). https://doi.org/10.1140/epje/i200101169

[37] Bormashenko E., Pogreb R., Musin A., Stanevsky O., Bormashenko Y., Whyman G., Gendelman O., Barkay Z.: Self-assembly in evaporated polymer solutions: Influence of the solution concentration. Journal of Colloid and Interface Science, 297, 534-540 (2006).

https://doi.org/10.1016/j.jcis.2005.11.025

[38] Bormashenko E., Pogreb R., Stanevsky O., Bormashenko Y., Stein T., Gengelman O.: Mesoscopic patterning in evaporated polymer solutions: New experimental data and physical mechanisms. Langmuir, 21, 9604-9609 (2005).

https://doi.org/10.1021/la0518492

[39] Sun W., Yang F.: Formation of self-organized gradient stripes on precast poly(methyl methacrylate) films. Langmuir, 30, 6548-6555 (2014). https://doi.org/10.1021/1a5004275

[40] Hong S. W., Xia J., Lin Z.: Spontaneous formation of mesoscale polymer patterns in an evaporating bound solution. Advanced Materials, 19, 1413-1417 (2007). https://doi.org/10.1002/adma.200601882

[41] Bormashenko E.: Breath-figure self-assembly, a versatile method of manufacturing membranes and porous structures: Physical, chemical and technological aspects. Membranes, 7, 45/1-45/20 (2017). https://doi.org/10.3390/membranes7030045 